\title{
Anomalous aortic origin of a coronary artery: The gaps and the guidelines
}

\author{
Marshall L. Jacobs, MD
}

From the Division of Cardiac Surgery, The Johns Hopkins Medical Institutions, Baltimore, Md.

Disclosures: Author has nothing to disclose with regard to commercial support.

Received for publication July 27, 2016; accepted for publication July 29, 2016; available ahead of print Aug 30, 2016.

Address for reprints: Marshall L. Jacobs, MD, Division of Cardiac Surgery, The Johns Hopkins Hospital, Zayed

7107, 1800 Orleans St, Baltimore, MD 21287 (E-mail: marshall.jacobs@comcast.net).

J Thorac Cardiovasc Surg 2017;153:1462-5

0022-5223/\$36.00

Copyright (C) 2016 by The American Association for Thoracic Surgery

http://dx.doi.org/10.1016/j.jtcvs.2016.07.058

For many cardiac surgeons, anomalous aortic origin of a coronary artery (AAOCA) first "crossed the radar screen" as the subject of a 1974 report by Dr Melvin Cheitlin and associates at Walter Reed Army Medical Center, titled "Sudden Death as a Complication of Anomalous Left Coronary Origin From the Anterior Sinus of Valsalva, a NotSo-Minor Congenital Anomaly." ${ }^{1}$ As the not-so-subtle title infers, the report debunked a then prevalent perception that origin of both coronary arteries from the same sinus of Valsalva was a minor coronary anomaly of little clinical significance, thought to be compatible with a long and active life. The report described what was probably the first successful surgical "unroofing" procedure, although the immediate objective had simply been to enlarge the slit-like orifice of the left coronary artery. Cheitlin and associates reviewed the entire collection of cases within the Armed Forces Institute of Pathology in which coronary artery anomalies had been identified. There were 33 patients in whom both coronary arteries arose from the anterior sinus of Valsalva and 18 patients in whom both coronaries arose from the left sinus of Valsalva. Of the 33 patients with anomalous aortic origin of the left coronary artery (AAOLCA) from the anterior (right) sinus, 9 had sudden, unexplained death. Seven of the 9 patients died during or immediately after exercise. Of 18 patients in whom both coronaries arose from the left sinus of Valsalva (anomalous aortic origin of the right coronary artery [AAORCA]), all had died of known, unrelated causes.

The implication of Cheitlin and colleagues' report ${ }^{1}$ was that AAOLCA with an interarterial course was a potentially malignant clinical entity that predisposed patients to lethal ischemic events. This initial focus, limited to AAOLCA, eventually gave way to a broader appreciation of the potential risks associated with anomalies of origin of either coronary artery, as other autopsy series then identified cases in which AAORCA had been associated with sudden cardiac death. ${ }^{2}$ For a time, efforts concentrated on estimating the risk of sudden death secondary to AAOCA in competitive athletes, relying primarily on data from autopsy series. ${ }^{3}$ A complex picture emerged that described an important risk

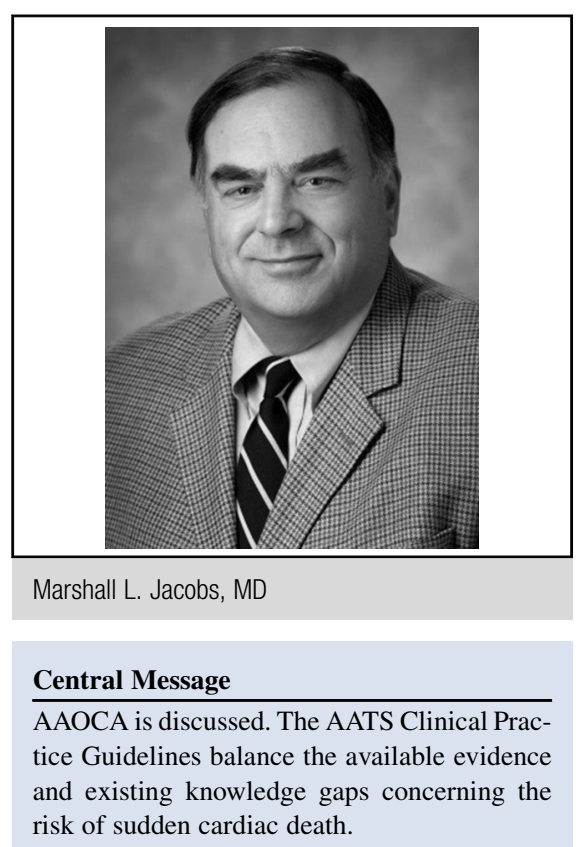

See Article page 1440 .

of sudden cardiac death among individuals with AAOCA, usually during or just after physical exertion. It became apparent that these lethal events were more numerous in patients with AAOLCA, although we eventually learned that AAOLCA was actually less common than AAORCA. Numerous possible etiologies of sudden cardiac death during or after exercise were proposed: further restriction of an already slit-like orifice by increased aortic pressure, kinking of the sharp angulation at the aortic take-off of the coronary artery, increased aortic pressure causing distortion or compression of an intramural segment, "squeezing" of an interarterial segment by the aorta and pulmonary artery, and spasm of the coronary artery. Of course, the information gleaned from autopsy series provided some information on the prevalence and correlates of sudden cardiac death, but only among those who had already died! It was not particularly useful in estimating the risk of death for those living with AAOCA.

Surgical strategies were proposed and applied to rescue those patients with aborted sudden death episodes. These operations were extended next to patients who experienced symptoms thought to be consistent with myocardial ischemia. Finally, they were extended to some asymptomatic patients in an effort to change a "natural history" of 
AAOCA that was incompletely understood, but certainly disconcerting for some. Our growing awareness of AAOCA and its clinical implications reflected a process of acquiring new knowledge that periodically shattered old myths. We learned early on that the "evidence-based" axiom that AAOLCA was an anatomic substrate for sudden cardiac death but AAORCA was not was incorrect. As mentioned, a review of additional autopsy series revealed instances of sudden death in patients with AAORCA. Also, an expanding portfolio emerged of case reports describing aborted sudden cardiac death among individuals with AAORCA from the left coronary sinus. In some of these, sudden cardiac death was the first symptom. ${ }^{4-6}$ As with most things in medicine, new evidence led to new, or at least changing, inferences. Yet when investigators began to seek the elusive denominator (ie, how many people are there with AAOCA?), it became evident that many patients with AAOCA live to a ripe old age without adverse cardiac events, including many with AAOLCA. What is it that distinguishes these patients from the unfortunate subset of those with AAOLCA who experience ischemic events early in life?

Over 3 decades, efforts to codify and analyze the available evidence have led to the development and periodic updating of guidelines for the evaluation and management of patients with congenital coronary anomalies. At first, these pertained primarily to the perceived risk of participating in competitive sports. Initially, restrictions were recommended only for patients with AAOLCA with an interarterial course. Subsequently, the recommended restrictions were extended to patients with AAOLCA or AAORCA, without distinction. Finally, the most recent scientific statement from the American Heart Association and American College of Cardiology emphasizes the distinction between those with AAOLCA (perceived to be at greatest risk) and those with AAORCA (lesser risk), and a further distinction between symptomatic patients with AAORCA and those without symptoms or a positive exercise stress test. ${ }^{7}$ Of note, those guidelines include no explicit recommendations regarding surgical repair. But a role for surgery is implicitly inferred, because the guidelines do suggest that the restrictions on physical activity, when applicable, should be enforced "before a surgical repair." And they go on to suggest that "after successful surgical repair of an anomalous origin from the wrong sinus, athletes may consider participation in all sports 3 months after surgery if the patient remains free of symptoms and an exercise stress test shows no evidence of ischemia or cardiac arrhythmias." 7

Most recently, The American Association for Thoracic Surgery (AATS) assembled a dedicated multidisciplinary expert panel to undertake a systematic literature review and develop evidence-based guidelines for the management of AAOCA. ${ }^{8}$ By using methodology consistent with standards put forth by the Institute of Medicine, the Writing
Committee summarized the literature, presented a comprehensive bibliography, and proposed recommendations that are broken down into those pertaining to diagnostic imaging, treatment, and follow-up. Of the 7 "Recommendations on Treatment," 4 are based on evidence level B and 3 are based on evidence level C. The recommendation that all patients with AAOCA and symptoms of ischemic chest pain, or syncope suspected to be due to ventricular arrhythmias, or a history of aborted sudden cardiac death should have activity restricted and be offered surgery is consistent with current patterns of practice at many centers. But the recommendation that individuals without symptoms with AAOLCA from the right sinus of Valsalva with an interarterial course, without further qualification, should be offered surgery is one that goes beyond the pattern of surgical referral at some centers where patients with AAOLCA who are without symptoms and have a negative stress test are subjected to individualized plans of management that may or may not include referral for surgery. The Writing Committee's recommendations for the management of patients with AAORCA include referral for surgery for those with symptoms of ischemic chest pain, syncope suspected to be due to ventricular arrhythmias, or a history of aborted sudden cardiac death. But they recommend that for those without either symptoms concerning for ischemia or a positive exercise stress test, and after counseling concerning the risk of sudden cardiac death, participation in competitive athletics is permissible. For these individuals, consideration of surgical referral is not mentioned. This also differs from the patterns of practice at many centers, including some with specialized multidisciplinary programs for patients with congenital coronary anomalies. At one such center, the institutional approach has been to recommend surgical treatment for all patients identified with AAOCA between the ages of 10 and 30 years, with a more selective approach based on symptoms and other factors for patients aged less than 10 years or more than 30 years. ${ }^{9}$ At another center with a dedicated coronary anomalies program, the approach to patients with AAORCA differs from the guideline recommendations in that patients without symptoms ascribed to ischemia and without a positive exercise stress test or radionuclide perfusion study nonetheless may be considered for surgery on the basis of additional considerations. These include high-risk anatomy (imaging findings of intramural course, abnormal ostium, dynamic changes of ostium and proximal course during the cardiac cycle) or coronary vessel dominance, desire to participate in competitive sports, or significant family anxiety. ${ }^{10}$

Here is where the rubber meets the road. What are the implications of institutional practices that deviate from those recommended in guidelines from professional societies? Some have argued that it is not the intended purpose of guidelines to take the place of clinical judgment and personalized care, but rather to provide a foundation that is a 
starting point for clinical decision making. This important distinction was emphasized by AATS President Thoralf Sundt in a recent editorial titled "Guidelines or Gospels?" 11 On the other hand, publication of clinical guidelines by professional societies carries considerable weight and may increase the onus and burden on individual practitioners or teams whose clinical experience, knowledge of the individual patient, and best judgment may draw them toward recommending a different course of management than that espoused in a published guideline. Management of individual patients with AAOCA is essentially a matter of arriving at a best estimate of the likelihood of ischemia leading to sudden cardiac death and trying to determine for each specific patient whether an intervention that is intended to alter the natural history is likely to mitigate a significant risk without imposing new and potentially greater ones. Forty years after the potential importance of these congenital cardiac anomalies began to become apparent, we still have an incomplete understanding of mechanisms of sudden cardiac death and the risk of sudden cardiac death associated with each of the different anomalies of coronary origin. There is little certainty about the potential importance of individual patient factors, including details of morphology that may differ from one case to the next. As importantly, the potential for both mitigation of risk and possible late sequelae associated with the different management strategies remains incompletely understood, particularly over the long term.

The AATS AAOCA Guideline Writing Committee offered the following assertion with regard to the risk of sudden death: "Despite not knowing the exact mechanism of ischemia in those with AAOCA, identifying and treating those anomalies that place the patient at risk of sudden cardiac death is of utmost importance and management should ideally be based on the risk assessment." ${ }^{8}$ This seems eminently logical. At the same time, the Writing Committee acknowledges the existence of major knowledge gaps regarding estimation of risk. Referring to the asymptomatic young patient with AAORCA, they say, "While there are suspected anatomic and physiologic reasons for myocardial ischemia and/or potentially lethal arrhythmias, we are still unable to distinguish which person is at high risk for ischemia and should undergo surgery from the individual who will remain asymptomatic and may be allowed to participate in competitive sports. ${ }^{\circ}$ This uncertainty with regard to risk stratification is one important knowledge gap. Another relates to the limited information available concerning patient status at long-term follow-up after conservative management or surgical intervention. Thus, it is difficult to know, on a case-by-case basis, whether the unnatural (postsurgical) history is likely to be preferable to the natural history of the disease in patients with all but the most high-risk categories of AAOCA.
Currently, there are few answers to questions regarding risk stratification in relation to factors more granular than right versus left and symptomatic versus asymptomatic. But the likelihood of answering such questions may be greater if data and experience from multiple institutions are pooled for analysis. There are no shortcuts to lessons learned from longitudinal follow-up. The Congenital Heart Surgeons' Society AAOCA multi-institutional registry represents an effort to address some of the important knowledge gaps. The Registry has now collected data on more than 500 patients with AAOCA. The investigators are in the early stages of a subcohort analysis focusing on patients who have had well-documented episodes of myocardial ischemia (J.A. Brothers, M.L. Jacobs, J.K. Kirklin, W. DeCampli, W.G. Williams, personal communication, June 8, 2016). This includes, but is not limited to, episodes of aborted sudden cardiac death. Of more than 500 patients in the registry, only approximately $2 \%$ have had aborted sudden death episodes. Of those $2 \%$, patients with AAOLCA outnumber those with AAORCA, but only by approximately 2 to 1 . When one considers all patients who have had documented ischemia (those with aborted sudden death, those with symptoms and documented ischemia, and those with ischemia during stress testing), they make up only approximately $5 \%$ to $6 \%$ of the registry. They are roughly evenly divided between AAORCA and AAOLCA. These preliminary figures will require review and validation. At that point, an effort will be made to codify as much data as possible concerning the clinical history, details of morphology based on echocardiographic and axial imaging (computed tomography and magnetic resonance imaging), and provocative stress testing. Potentially relevant details of morphology include not only identification of the coronary artery with anomalous origin but also ostial shape and cross-sectional area, proximal coronary angulation, interarterial length, intramural length, maximal and minimal cross-sectional areas, area stenosis, and degree of ellipticity (coronary arterial height and width) of interarterial and intramural segments. ${ }^{12}$ For those who have undergone surgery, details of the operative findings will be included in the analysis. A first tier of analysis will be undertaken evaluating which morphologic elements, if any, are shared by many of the patients who have had ischemia. Ultimately, it will be necessary to see how these details and measurements of morphology compare with patients who have not experienced ischemia, with either normal activity or provocative testing. The hope is that it will be possible to stratify risk on the basis of more information than simply which artery has anomalous origin and whether or not the patient has exhibited compelling symptoms. The hypothesis is that some details of morphology, or combinations of details, ultimately may be shown to be associated with an increased risk of ischemia. 
The body of available information about AAOCA grows constantly. Periodic systematic review is essential. In the past, with respect to AAOCA, most multicentric data analyses have been limited to information pertaining to patients who have died. Most of the analyses of data pertaining to living patients are from individual centers and reflect their patterns of referral and management. The AATS Writing Committee has carefully analyzed all of the currently available published material. Their report adds clarity to a complex field of information and renders much of the information accessible in one place. The authors are candid about important knowledge gaps and their implications for recommendations they offer. Their recommendations with respect to treatment reflect their careful analysis of available information regarding the risk of sudden cardiac death in patients with AAOCA and their shared expert opinions regarding the quality of available evidence and best practices in light of that evidence. These recommendations have an important place in the current mixture of guidelines, gospels, and gaps.

\section{References}

1. Cheitlin MD, De Castro CM, McAllister HA. Sudden death as a complication of anomalous left coronary origin from the anterior sinus of Valsalva, A not-sominor congenital anomaly. Circulation. 1974;50:780-7.
2. Frescura C, Basso C, Thiene G, Corrado D, Pennelli T, Angelini A, et al. Anomalous origin of coronary arteries and risk of sudden death: a study based on an autopsy population of congenital heart disease. Hum Pathol. 1998;29:689-95.

3. Basso C, Maron BJ, Corrado D, Thiene G. Clinical profile of congenital coronary artery anomalies with origin from the wrong aortic sinus leading to sudden death in young competitive athletes. J Am Coll Cardiol. 2000;35:1493-501.

4. Yanagawa B, Alghamdi AA, Chen RB, Amankwaa A, Verma SJ. Coronary artery bypass graft for anomalous right coronary artery. J Card Surg. 2011;26:44-6.

5. Muñoz-Guijosa C, Permanyer E, Leta R. Anomalous origin of right coronary artery from the left coronary sinus: sudden death and successful surgical reimplantation. Eur Heart J. 2012;33:1308.

6. Elhmidi Y, Nöbauer C, Hörer J, Schreiber C, Lange R. Surgical unroofing of an anomalous right coronary artery arising from the posterior left sinus of Valsalva. World J Pediatr Congenit Heart Surg. 2013;4:433-5.

7. Van Hare GF, Ackerman MJ, Evangelista JA, Kovacs RJ, Myerburg RJ, Shafer KM, et al. Eligibility and Disqualification Recommendations for Competitive Athletes With Cardiovascular Abnormalities: Task Force 4: Congenita Heart Disease: A Scientific Statement From the American Heart Association and American College of Cardiology. J Am Coll Cardiol. 2015;66:2372-84.

8. Brothers JA, Frommelt MA, Jaquiss RDB, Myerburg RJ, Fraser CD, Tweddell JS. Expert consensus guidelines: Anomalous aortic origin of a coronary artery. J Thorac Cardiovasc Surg. 2017;153:1440-57.

9. Mainwaring RD, Murphy DJ, Rogers IS, Chan FP, Petrossian E, Palmon M, et al. Surgical repair of 115 patients with anomalous aortic origin of a coronary artery from a single institution. World J Pediatr Congenit Heart Surg. 2016;7:353-9.

10. Mery CM, Lawrence SM, Krishnamurthy R, Sexson-Tejtel SK, Carberry KE McKenzie ED, et al. Anomalous aortic origin of a coronary artery: toward a standardized approach. Semin Thorac Cardiovasc Surg. 2014;26:110-22.

11. Sundt TM. Guidelines or gospels? J Thorac Cardiovasc Surg. 2016;151:1472-4.

12. Lee S, Uppu SC, Lytrivi ID, Sanz J, Weigand J, Geiger MK, et al. Utility of multimodality imaging in the morphologic characterization of anomalous aortic origin of a coronary artery. World J Pediatr Congenit Heart Surg. 2016;7:308-17. 